\title{
Estructura poblacional de Teline monspessulana (L.) K. Koch en fragmentos de bosque maulino en Chile central
}

\section{Population structure of Teline monspessulana (L.) K. Koch in fragments of maulino forest in Central Chile}

\author{
Persy Gómez ${ }^{1}$, Ramiro Bustamante ${ }^{2,3}$ \& José San Martin ${ }^{4}$ \\ 1Jardín Botánico, Universidad de Talca, Casilla 747, Talca, Chile. \\ ${ }^{2}$ Facultad de Ciencias, Universidad de Chile, Casilla 653, Santiago, Chile. \\ ${ }^{3}$ Instituto de Ecología y Biodiversidad (IEB), Facultad de Ciencias, Universidad de Chile, Casilla 653, Santiago, Chile. \\ ${ }^{4}$ Instituto de Biología Vegetal y Biotecnología, Universidad de Talca, Casilla 747, Talca, Chile. \\ persy_gomez@hotmail.com
}

\begin{abstract}
In this note we examine the population structure of Teline monspessulana, an exotic plant that is invading fragments of the Maulino Forest. We evaluate density T. monspessulana, sizes, proportion of reproductive individuals and his relation with the size of the patches in 8 forest fragments. Our results indicate a high proportion of individuals growing inside fragments. Moreover, the fragment size is not correlated with T. monspessulana density.
\end{abstract}

En Chile, más del $60 \%$ de la flora total y de las especies endémicas se concentran en la zona central, área identificada como uno de los sitios del planeta prioritarios de conservar debido al alto número de endemismos y gran pérdida de hábitat (Myers et al. 2000). En esta zona se encuentra el Bosque Maulino, bosque templado dominado por especies endémicas de Nothofagus como Nothofagus glauca (Phil.) Krasser y Nothofagus alessandrii Espinosa, cuya superficie entre los años 1975 y 2000 se ha reducido en un 67\%. En su reemplazo, se han establecido plantaciones de Pinus radiata D.Don (Echeverría et al. 2006), generando un paisaje fragmentado con parches de vegetación nativa rodeados por estas plantaciones de pinos. Una de las consecuencias de la fragmentación de los bosques, es la llegada de especies exóticas (Krüger et al. 1989). Este proceso se relaciona con la influencia de la matriz sobre los parches, al facilitarse el proceso de colonización y los cambios en las condiciones internas que favorecen el establecimiento de estas especies (Pauchard et al. 2006). En este sentido, las interacciones entre atributos de los fragmentos (cobertura vegetal, régimen de disturbio, tamaño) y las características biológicas de las plantas exóticas (producción de semillas, mecanismos de dispersión, resistencia al fuego, habilidad competitiva), podrían dilucidar la capacidad potencial de las especies para invadir los fragmentos (Brothers \& Spingarn 1992, Laurance 1997, Viana et al. 1997, Standish et al. 2001).

Una especie introducida perteneciente a la familia Fabaceae, altamente invasiva en la zona de clima mediterráneo y parte del territorio con clima templado de
Chile, es Teline monspessulana (L.) K. Koch (García et al. 2007, 2010, Zuloaga et al. 2008), especie que también es posible encontrarla en zonas climáticamente similares con frecuencia de incendios (Australia, California), o bien en otras regiones con climas diferentes (Canadá, Nueva Zelanda y EE.UU.) (Adams \& Simmons 1991, Bossard 2000, García et al. 2010).

Actualmente en Chile, T. monspessulana crece formando poblaciones naturalizadas entre la V Región de Valparaíso y la X Región de los Lagos (Zuloaga et al. 2008). Se encuentra frecuentemente en claros en el sotobosque de las plantaciones de P. radiata y Eucalyptus globulus Labill., y en áreas abiertas o degradadas formando extensos matorrales (García et al. 2007). Además, es abundante en zonas húmedas y suelos ácidos y arenosos, orillas de caminos y sitios perturbados en áreas cordilleranas. En California, Australia y Nueva Zelanda es considerado uno de los arbustos invasores más severos (Bossard 2000, Alexander \& D'Antonio 2003a). Por otra parte, las semillas de $T$. monspessulana poseen una testa dura y pueden permanecer sin germinar durante años, generando bancos de semillas persistentes bajo las plantas madres. Esta capacidad asegura la regeneración masiva de la especie luego de incendios, razón por la cual es considerada una maleza de difícil control (Alexander \& D'Antonio 2003b, García et al. 2010). En Chile, la dinámica de regeneración y posterior establecimiento de T. monspessulana y su relación con la ocurrencia de perturbaciones ha sido poco estudiada, pero la evidencia empírica demuestra que está ligada a incendios 
forestales (García et al. 2010). En este marco, y en un paisaje fragmentado es relevante examinar las características de las poblaciones de esta especie introducida de modo de inferir la magnitud de este proceso invasivo. Los objetivos de esta investigación fueron determinar la estructura poblacional de T. monspessulana y evaluar la correlación entre el tamaño de los fragmentos de bosque maulino y la densidad de $T$. monspessulana. El estudio se desarrolló en la comuna de Pelluhue, provincia de Cauquenes, Región del Maule, Chile central, y en donde se evaluó la densidad poblacional, la estructura de tamaños y la proporción de individuos reproductivos; además estas variables se correlacionaron con el tamaño de los parches, ya que en la teoría la disminución en la superficie de los fragmentos se asocia a un incremento del efecto de borde, favoreciendo la llegada de especies invasoras (Aizen \& Feisinger 1994).

Se trabajó en época estival en ocho fragmentos dominados por la especie Nothofagus glauca (Phil.) Krasser, cuyas superficies varían entre las 3 y las 152 ha (Fig.1). Se realizaron 162 cuadrantes de $10 \times 10 \mathrm{~m}$, distribuidos desigualmente en los 8 fragmentos (Tabla I). En cada cuadrante se contabilizaron todos los individuos de T. monspessulana. Además, se determinó el estado juvenil o reproductivo de los individuos, registrando como reproductivos todos aquellos que presentaban frutos.

Para la superficie muestreada $\left(16.200 \mathrm{~m}^{2}\right)$ en los ocho fragmentos de bosque maulino se encontró una abundancia total de 893 individuos de T. monspessulana (Tabla I), distribuidos principalmente en sitios abiertos o con escasa cobertura del dosel. El 94,5 \% de los individuos fueron juveniles, en cambio los reproductivos fueron en total 76 individuos (8,5\%) (Fig. 2). No se encontraron individuos de G. monspessulana en el fragmento 3 (Tabla I), fragmento de menor tamaño y que fisonómicamente presentó la menor cobertura vegetal. En cambio el fragmento 6 presentó una abundancia muy sobre la media, desconociendo que atributos de este fragmento pueden servir como principales indicadores de invasibilidad. A partir de los $60 \mathrm{~cm}$ de altura es posible encontrar individuos reproductivos.

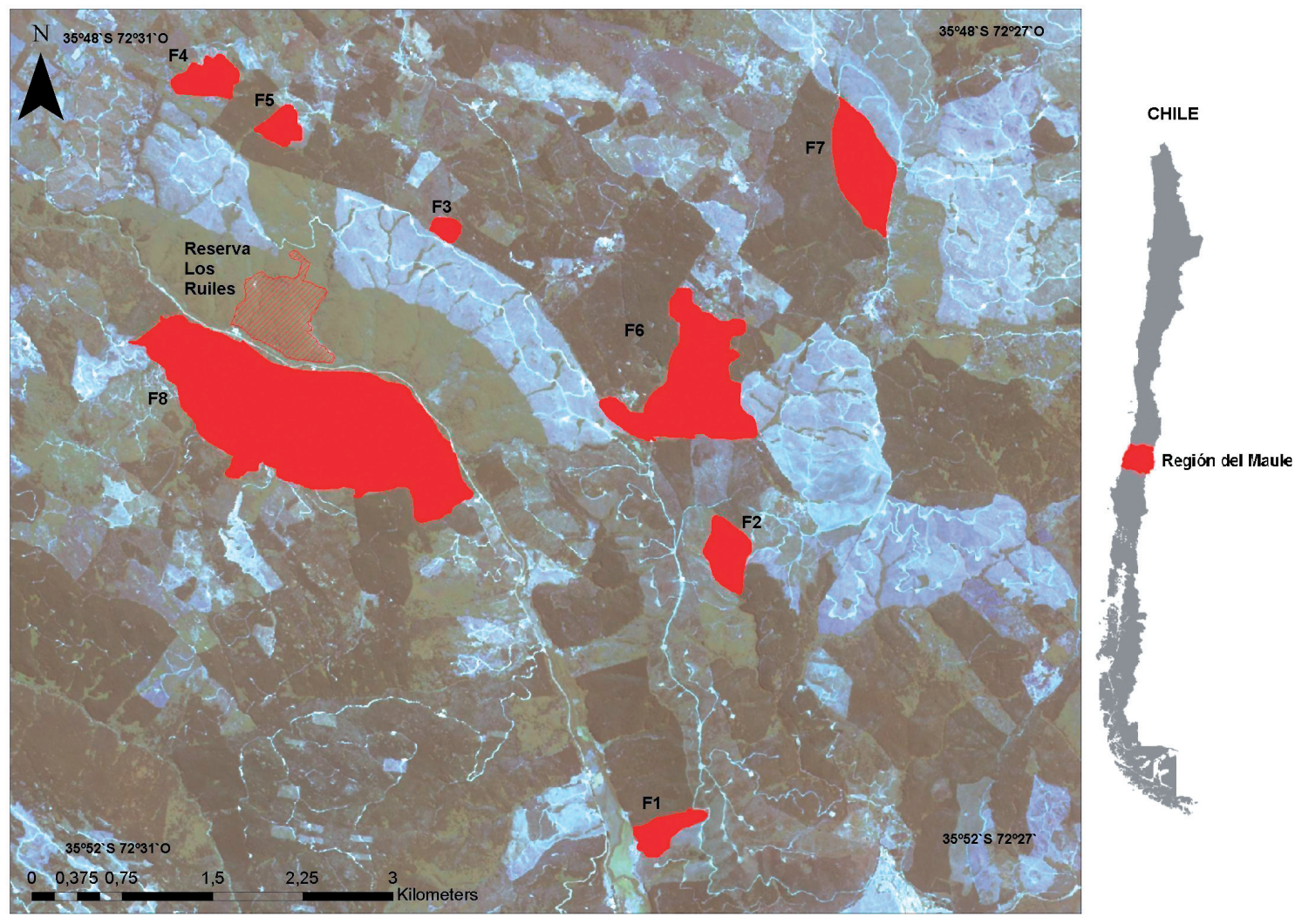

Figura 1. Distribución espacial de los fragmentos de Bosque Maulino, Provincia de Cauquenes, Región del Maule, Chile (F=fragmento).

Figure 1. Spatial distribution of Maulino forest fragments, Provincia de Cauquenes, Maule Region, Chile ( $\mathrm{F}=$ fragment). 
Estructura poblacional de Teline monspessulana: GómEz, P. ET AL.

Tabla I. Tamaño de los fragmentos y abundancia total de Teline monspessulana en ocho fragmentos de Bosque Maulino, Provincia de Cauquenes, Región del Maule, Chile.

TABLE I. Fragment size and Teline monspessulana total abundance across eight fragments of Maulino Forest, Provincia de Cauquenes, Maule Region, Chile.

\begin{tabular}{cccc}
\hline Fragmento & $\begin{array}{c}\text { SuperficIE } \\
(\mathrm{ha})\end{array}$ & $\begin{array}{c}\text { SuperficIE } \\
\text { MUESTREADA }\left(\mathrm{m}^{2}\right)\end{array}$ & $\begin{array}{c}\text { AbUNDANCIA TOTAL } \\
\text { EN SUPERFICIE } \\
\text { MUESTREADA }\end{array}$ \\
\hline 1 & 11 & 1100 & 26 \\
2 & 19 & 1500 & 48 \\
3 & 3 & 300 & 0 \\
4 & 20 & 1600 & 49 \\
5 & 7 & 700 & 14 \\
6 & 70 & 3500 & 638 \\
7 & 53 & 3000 & 83 \\
8 & 152 & 4500 & 35 \\
Total & 335 & 16.200 & 893 \\
\hline
\end{tabular}

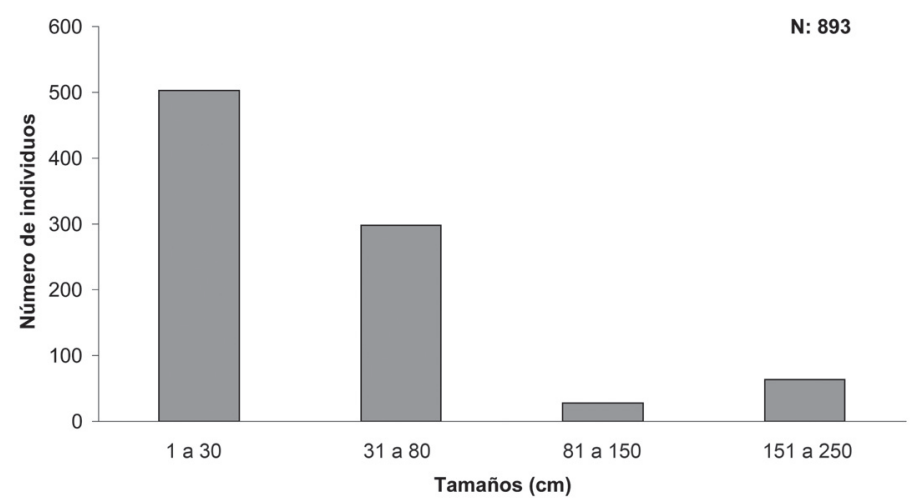

Figura 2. Estructura de alturas de Teline monspessulana en los ocho fragmentos de Bosque Maulino, Provincia de Cauquenes, Región del Maule, Chile.

Figure 2. Heights structure of Teline monspessulana in eight fragments of Maulino Forest, Provincia de Cauquenes, Maule Region, Chile.

La densidad de T. monspessulana no se correlacionó con el tamaño de los fragmentos (Rho de Spearman 0,4045, p $>0,01)$.

De estos resultados se concluye que el proceso de invasión de T. monspessulana a los fragmentos de Bosque Maulino es un fenómeno reciente, así lo indican la gran cantidad de individuos jóvenes y la escasez de individuos reproductores. La presencia de $T$. monspessulana en el Bosque Maulino puede ser producto de un efecto conjunto de semillas que llegan desde la matriz circundante, más las producidas por los individuos reproductores de la especie ya establecidos. Se desconoce si la gran cantidad de individuos jóvenes de T. monspessulana presentes en los parches serán capaces de sobrevivir y establecerse. A nivel de micrositio, bajas coberturas del dosel no aseguran una alta abundancia de individuos de T. monspessulana como se observa en el fragmento 3. Para el área de estudio, el corto periodo juvenil de la especie (individuos reproductivos a partir de los 60 $\mathrm{cm}$ de altura) incrementa la probabilidad de invasión. El tamaño del fragmento no parece ser un buen indicador de la susceptibilidad del Bosque Maulino a ser invadido por esta especie. T. monspessulana se encuentra invadiendo los fragmentos de Bosque Maulino, desconociendo aún sus consecuencias, por lo cual urge la necesidad de evaluar la influencia de variables ambientales y de paisaje en el reclutamiento de esta especie, con el fin de poseer mayores herramientas que ayuden a la toma de decisiones y manejo del Bosque Maulino. 


\section{AGRADECIMIENTOS}

Se agradece al proyecto "Estructura de redes mutualistas en bosques fragmentados". Anillo de Investigación en Ciencia y Tecnología, PBCT, Chile.

\section{BIBLIOGRAFIA}

Adams, R. \& D. Simmons. 1991. The invasive potential of Genista monspessulana (Montpellier broom) in dry sclerophyll forest in Victoria. Victorian Naturalist 108(4): 84-89.

Aizen, M.A. \& P. FeIsINGER. 1994. Forest fragmentation, pollination and plant reproduction in a Chaco dry forest, Argentina. Ecology 75: 330-351.

Alexander, J. \& C.M. D’Antonio. 2003a. Seed bank dynamics of French broom in coastal California grasslands: Effects of stand age and prescribed burning on control and restoration. Restoration Ecology 11: 185-197.

Alexander, J. \& C.M. D'Antonio. 2003b. Control methods the removal of French and Scotch Broom tested in coastal California. Ecological Restoration 21: 191-198.

Bossard, C. 2000. Genista monspessulana (L.) L. Johnson. In: C.C. Bossard, J.M. Randall \& M.C. Hoshovsky (eds.), Invasive plants of California's wildlands: 203-208. University of California Press, Berkeley, USA.

Brothers, T.S. \& A. Spingarn. 1992. Forest fragmentation and alien plant invasion of central indiana old-growth forests. Conservation Biology 6: 91-100.

Echeverría, C., A. Newton, A. Lara, JM. Rey-Benayas \& D. CoOMEs. 2006. Impacts of forest fragmentation on species composition and forest structure in the temperate landscape of southern Chile. Global Ecology and Biogeography 16: 426-439.

García, R.A., A. Pauchard \& E. Peña. 2007. Invasión de Teline monspessulana (L.) K. Koch después de un incendio forestal: Banco de semillas, regeneración y crecimiento. Gayana Botánica 64: 200-209.

García, R.A., A. Pauchard, L. Cavieres, E. Peña \& M. RodríGUEZ. 2010. El fuego favorece la invasión de Teline monspessulana (Fabaceae) al aumentar su germinación.
Revista Chilena de Historia Natural 83: 443-452.

KrÜGer, F.J., G.J. Breytenbach, I.A.W. MacDonald \& D.M. RICHARDSON. 1989. The characteristics of invaded Mediterranean climate regions. In: J.A. Drake, H.A. Mooney, F. di Castri, R.H. Groves, F.J. Krüger, M. Rejmánek \& M. Williamson (eds.), Biological Invasions: a global perspective, pp. 181-213. Wiley, Chichester, UK.

LaURANCE, W.F. 1997. Hyper-disturbed parks: edge effects and the ecology of isolated reserves in tropical Australia. In: W.F. Laurance \& R.O. Bierregaard (eds.), Tropical Forest Remmants: Ecology, Management and Conservation of fragmented communities. University of Chicago Press, Chicago, pp. 71-84.

Myers, N., R.A. Mittermeier, C.G. Mittermeier, G.A.B. dA FonseCA \& J. Kent. 2000. Biodiversity hotspots for conservation priorities. Nature 403: 853-858.

Pauchard, A., M. Aguayo \& P. Alaback. 2006. Cuantificando la fragmentación del paisaje: las métricas y sus significados ecológicos. En: A. Grez, J. Simonetti \& R.O. Bustamante (eds.), Biodiversidad en ambientes fragmentados de Chile: patrones y procesos a diferentes escalas, pp. 41-67. Editorial Universitaria, Santiago, Chile.

Richardson, D.M., P. Pysek, M. Rejmánek, M.G. Barbour, F.D. PanetT \& C.T. West. 2000. Naturalization and invasion of alien plant: concepts and definitions. Diversity and Distributions 6: 93-107.

Standish, R.J., A.W. Robertson \& P.A. Williams. 2001. The impact of an invasive weed Tradescantia fluminensis on native forest regeneration. Journal of Applied Ecology 38: 1253-1263.

Viana, V.M., A.A.J. Tabanez \& J.L. Batista. 1997. Dynamics and restoration of forest fragments in Brazilian Atlantic moist forest. In: W.F. Laurance \& R.O. Bierregaard (eds.), Tropical Forest Remmants: Ecology, Management and Conservation of fragmented communities. University of Chicago Press, Chicago, pp. 351-365.

Zuloaga, F.O., O. Morrone \& M. Belgrano. 2008. Catálogo de las plantas vasculares del Cono Sur. Volumen 2: Dicotyledoneae: Acanthaceae-Fabaceae (AbaremaSchizolobium). Monographs in Systematic Botany 107, Missouri Botanical Garden Press, St. Louis, Missouri, USA. 985-2286 pp. 